\title{
EQUIPE EDITORIAL E AVALIADORES AD HOC - VOLUME 8, N. 2, DA REVISTA REAMEC
}

\section{Editora-chefe / Diretora}

Dra. Gladys Denise Wielewski, Universidade Federal de Mato Grosso (UFMT), Programa de Pós-Graduação em Educação em Ciências e Matemática (PPGECEM) da Rede Amazônica de Educação em Ciências e Matemática (REAMEC), Cuiabá, Mato Grosso, Brasil.

\section{Editores Associados / Corpo Editorial}

Dr. Cristiano da Silva Macêdo, Instituto Federal do Maranhão (IFMA), Timon, Maranhão, Brasil.

Me. Dailson Evangelista Costa, Universidade Federal do Tocantins (UFT), Arraias, Tocantins, Brasil.

Dra. Josefina Barrera Kalhil, Universidade do estado do Amazonas (UEA), Manaus, Amazonas, Brasil.

Dr. Licurgo Peixoto de Brito, Universidade Federal do Pará (UFPA), Belém, Pará, Brasil.

Dr. Marcel Thiago Damasceno Ribeiro, Universidade Federal de Mato Grosso (UFMT), Cuiabá, Mato Grosso, Brasil.

Me. Thiago Beirigo Lopes, Instituto Federal de Mato Grosso (IFMT), Confresa, Mato Grosso, Brasil.

\section{Equipe Técnica}

Me. Dailson Evangelista Costa, Universidade Federal do Tocantins (UFT), Arraias, Tocantins, Brasil.

Me. Luis Andrés Castillo B., Aprender en Red (AR), Maracaibo, Zulia, Venezuela.

Me. Thiago Beirigo Lopes, Federal de Mato Grosso (IFMT), Confresa, Mato Grosso, Brasil.

\section{Conselho Científico Consultivo (Nacional)}

Dr. Ademir de Souza Pereira, Universidade Federal da Grande Dourados (UFGD), Dourados, Mato Grosso do Sul, Brasil.

Dra. Ana Carolina Araújo da Silva, Universidade Federal de Juiz de Fora (UFJF), Juiz de Fora, Minas Gerais, Brasil.

Dr. Bruno Silva Leite, Universidade Federal Rural de Pernambuco (UFRPE), Recife, Pernambuco, Brasil.

Dr. David Antonio da Costa, Universidade Federal de Santa Catarina (UFSC), Florianópolis, Santa Catarina, Brasil.

Dr. Geison Jader Mello, Instituto Federal de Mato Grosso (IFMT), Cuiabá, Mato Grosso, Brasil.

Dr. Geraldo Eustáquio Moreira, Universidade de Brasília (UnB), Brasília, Distrito Federal, Brasil.

Dr. Idemar Vizolli, Universidade Federal do Tocantins (UFT), Palmas, Tocantins, Brasil.

Dr. José Roberto Linhares de Mattos, Universidade Federal Fluminense (UFF), Niterói, Rio de Janeiro, Brasil. 
Dr. José Sávio Bicho, Universidade Federal do Sul e Sudeste do Pará (UNIFESSPA), Marabá, Pará, Brasil.

Dr. Leandro Carbo, Instituto Federal de Mato Grosso (IFMT), Jaciara, Mato Grosso, Brasil. Dra. Maria Alice de Vasconcelos Feio Messias, Universidade do Estado do Pará (UEPA), Belém, Pará, Brasil.

Dr. Nelson Antonio Pirola, Universidade Estadual Paulista (UNESP), Bauru, São Paulo, Brasil.

Dr. Thiago Henrique Barnabé Corrêa, Universidade Federal do Triângulo Mineiro (UFTM), Uberaba, Minas Gerais, Brasil.

Dr. Wellington Lima Cedro, Universidade Federal de Goiás (UFG), Goiânia, Goiás, Brasil.

\section{Conselho Científico Consultivo (Internacional)}

Dr. António Francisco Carrelhas Cachapuz, Universidade de Aveiro (UA), Aveiro, Portugal

Dr. Jesús Ángel Vila Muñoz, Universidad del País Vasco, Lejona, Espanha.

Dr. João Pedro Mendes da Ponte, Universidade de Lisboa (U-Lisboa), Lisboa, Portugal, Portugal.

Dr. José Carrillo-Yáñez, Universidad em la Universidad de Huelva (UHU), Huelva, España, Espanha.

\section{Comitê Científico Assessor (Nacional)}

Dr. Attico Inácio Chassot, Universidade Federal do Sul e Sudeste do Pará (UNIFESSPA), Marabá, Pará, Brasil.

Dr. Dario Fiorentini, Universidade Estadual de Campinas (UNICAMP), Campinas, São Paulo, Brasil.

Dr. Gerson Souza Mol, Universidade de Brasilia, Brasília, Distrito Federal, Brasil.

Dr. Iran Abreu Mendes, Universidade Federal do Pará (UFPA), Belém, Pará, Brasil.

Dra. Irene Cristina de Mello, Universidade Federal de Mato Grosso (UFMT), Cuiabá, Mato Grosso, Brasil.

Dra. Marta Maria Pontin Darsie, Universidade Federal de Mato Grosso (UFMT), Cuiabá, Mato Grosso, Brasil.

Dr. Milton Rosa, Universidade Federal de Ouro Preto (UFOP), Ouro Preto, Minas Gerais, Brasil.

Dra. Neuza Bertoni Pinto, Rede Amazônica de Educação em Ciências e Matemática (REAMEC), Cuiabá, Mato Grosso, Brasil.

Dra. Nyuara Araújo da Silva Mesquita, Universidade Federal de Goiás (UFG), Goiânia, Goiás, Brasil.

Dra. Roseli Pacheco Schnetzler, Universidade Federal do Pará (UFPA), Belém, Pará, Brasil. Dr. Tadeu Oliver Gonçalves, Universidade Federal do Pará (UFPA), Belém, Pará, Brasil.

Dr. Wagner Rodrigues Valente, Universidade Federal de São Paulo (UNIFESP), São Paulo, São Paulo, Brasil.

\section{Comitê Científico Assessor (Internacional)}

Dr. César Eduardo Mora Ley, Instituto Politécnico Nacional (IPN), Cidade do México, México.

Dra. Elizabeth Hernández Arredondo, Universidad de Los Lagos Chile (ULAGOS), Osorno, Chile. 
Dr. Enrique Arribas Gardes, Universidad de Castilla-La Mancha (UCM), Espanha.

Dr. Jaime Israel García-García, Universidad de Los Lagos (ULL), Osorno, Chile.

Dr. José Ortiz Buitrago, Universidad de Carabobo (UC), Maracay, Estado Aragua, Venezuela.

Dr. Mario Sánchez Aguilar, Instituto Politécnico Nacional (IPN), Ciudad de México, México.

Dr. Nelson Arias Ávila, Universidad Distrital Francisco José de Caldas (UDFJC), Bogotá, Colômbia.

\section{Avaliadores Ad Hoc}

1. Me. Adauto Nunes da Cunha, Instituto Federal de São Paulo (IFSP), Ilha Solteira, São Paulo, Brasil.

2. Dr. Adelmo Carvalho Silva, Universidade Federal de Mato Grosso (UFMT), Cuiabá, Mato Grosso, Brasil.

3. Dr. Ademir S. Pereira, Universidade Federal da Grande Dourados (UFGD), Dourados, Mato Grosso do Sul, Brasil.

4. Dra. Ana Carolina A. da Silva, Universidade Federal de Juiz de Fora, (UFJF), Juiz de Fora, Minas Gerais, Brasil.

5. Dra. Ana Paula Santos Rebello, Pontifícia Universidade Católica do Rio Grande do Sul (PUCRS), Porto Alegre, Rio Grande do Sul, Brasil.

6. Me. André Flávio Gonçalves Silva, Universidade Federal do Maranhão (UFMA), São Luís, Maranhão, Brasil.

7. Dra. Andreia Cristina Rodrigues Trevisan, Universidade Federal de Mato Grosso (UFMT), Sinop, Mato Grosso, Brasil.

8. Dra. Ataiany dos Santos Veloso Marques, Secretaria do Estado da Educação do Amazonas (SEDUC/AM), Paritins, Amazonas, Brasil.

9. Dra. Bianca Martins Santos, Universidade Federal do Acre (UFAC), Rio Branco, Acre, Brasil.

10. Me. Dailson Evangelista Costa, Universidade Federal do Tocantins (UFT), Arraias, Tocantins, Brasil.

11. Dr. David Antonio da Costa, Universidade Federal de Santa Catarina (UFSC), Florianópolis, Santa Catarina, Brasil.

12. Dr. Deive Barbosa Alves, Universidade Federal do Tocantins (UFT), Araguaína, Tocantins, Brasil.

13. Dr. Denivaldo Pantoja da Silva, Universidade Federal do Pará (UFPA), Cametá, Pará, Brasil.

14. Dr. Douglas Marin, Universidade Federal de Uberlândia (UFU), Uberlândia, Minas Gerais, Brasil.

15. Dra. Edileusa do Socorro Valente Belo, Universidade Federal de Roraima (UFRR), Boa Vista, Roraima, Brasil.

16. Dr. Ednilson Sergio Ramalho de Souza, Universidade Federal do Oeste do Pará (UFOPA), Santarém, Pará, Brasil.

17. Dr. Edson Pereira Barbosa, Universidade Federal de Mato Grosso (UFMT), Sinop, Mato Grosso, Brasil.

18. Dr. Eduardo José Cezari, Universidade Federal do Tocantins (UFT), Palmas, Tocantins, Brasil.

19. Dr. Eduardo Ribeiro Mueller, Universidade Federal de Mato Grosso (UFMT), Barra do Garças, Mato Grosso, Brasil. 
20. Dra. Elane Chaveiro Soares, Universidade Federal de Mato Grosso (UFMT), Cuiabá, Mato Grosso, Brasil.

21. Me. Eleonora Celli Carioca Arenare, Universidade do Estado do Amazonas (UEA), Boa do Acre, Amazonas, Brasil.

22. Dra. Eliane Regina Martins Batista, Universidade Federal do Amazonas (UFAM), Humaitá, Amazonas, Brasil.

23. Dra. Elisangela Dias Brugnera, Universidade do Estado de Mato Grosso (UNEMAT), Sinop, Mato Grosso, Brasil.

24. Dra. Elizabeth Cardoso Gerhardt Manfredo, Universidade Federal do Pará (UFPA), Belém, Pará, Brasil.

25. Me. Fábio Soares Pereira, Instituto Federal do Acre (IFAC), Sena Madureira, Acre, Brasil 26. Dra. Fatima Aparecida da Silva Iocca, Universidade do Estado de Mato Grosso (UNEMAT), Barra do Bugres, Mato Grosso, Brasil.

27. Dr. Francisco Pessoa Paiva Júnior, Instituto Federal do Maranhão (IFMA), Santa Inês, Maranhão, Brasil.

28. Me. Gahelyka Aghta Pantano Souza, Universidade Federal do Acre (UFAC), Rio Branco, Acre, Brasil.

29. Dra. Geovana Poton Arcobeli Cola, Instituto Federal do Espírito Santo (IFES), Alegre, Espírito Santo, Brasil.

30. Dra. Geslane Figueiredo da Silva Santana, Universidade Federal de Mato Grosso (UFMT), Sinop, Mato Grosso, Brasil.

31. Me. Gildemberg Cunha Silva, Instituto Federal do Tocantins (UFT), Araguaína, Tocantins, Brasil.

32. Dra. Glaucia Ribeiro Gonzaga, Universidade Federal Fluminense (UFF), Santo Antônio de Pádua, Rio de Janeiro, Brasil.

33. Dr. Hernani Luiz Azevedo, Universidade Federal de Mato Grosso (UFMT), Sinop, Mato Grosso, Brasil.

34. Dra. Ilma Maria Fernandes Soares, Universidade do Estado da Bahia (UNEB), Jacobina, Bahia, Brasil.

35. Dra. Irene Cristina de Mello, Universidade Federal de Mato Grosso (UFMT), Cuiabá, Mato Grosso, Brasil.

36. Dra. Irlane Maia Oliveira, Universidade Federal do Amazonas (UFAM), Manaus, Amazonas, Brasil.

37. Dra. Isabel do Socorro Lobato Beltrão, Universidade do Estado do Amazonas (UEA), Parintins, Amazonas, Brasil.

38. Dr. Italo Gabriel Neide, Universidade do Vale do Taquari (UNIVATES), Lajeado, Rio Grande do Sul, Brasil.

39. Me. Ivonne C. Sánchez S., Aprender en Red (AR), Maracaibo, Zulia, Venezuela.

40. Dra. Janeisi de Lima Meira, Universidade Federal do Tocantins (UFT), Arraias, Tocantins, Brasil.

41. Dr. Jean Carlos Miranda da Silva, Universidade Federal Fluminense (UFF), Santo Antônio de Pádua, Rio de Janeiro, Brasil.

42. Dr. João Cláudio Brandemberg Quaresma, Universidade Federal do Pará (UFPA), Belém, Pará, Brasil.

43. Dr. João Manoel da Silva Malheiros, Universidade Federal do Pará (UFPA), Castanhal, Pará, Brasil.

44. Dr. José Moysés Alves, Universidade Federal do Pará (UFPA), Belém, Pará, Brasil.

45. Dr. José Ricardo e Souza Mafra, Universidade Federal do Oeste do Pará (UFOPA), Santarém, Pará, Brasil. 
46. Dr. José Ronaldo Melo, Universidade Federal do Acre (UFAC), Rio Branco, Acre, Brasil.

47. Dr. José Sávio Bicho, Universidade Federal do Sul e Sudeste do Pará (UNIFESSPA), Marabá, Pará, Brasil.

48. Dra. Josefina Barrera Kalhil, Universidade do Estado do Amazonas (UEA), Manaus, Amazonas, Brasil.

49. Dr. Kaled Sulaiman Khidi, Universidade Federal do Tocantins (UFT), Arraias, Tocantins, Brasil.

50. Dra. Kátia Maria de Medeiros, Universidade Estadual da Paraíba (UEPB), Campina Grande, Paraíba, Brasil.

51. Dr. Leandro Carbo, Instituto Federal de Mato Grosso (IFMT), Jaciara, Mato Grosso, Brasil.

52. Dra. Lilian Cristiane A. dos Santos, Universidade Federal do Oeste do Pará (UFOPA), Santarém, Pará, Brasil.

53. Dra. Luciana da Cunha Ferreira, Universidade do Estado do Amazonas (UEA), Manaus, Amazonas, Brasil.

54. Me. Luis Andrés Castillo B., Universidade Federal do Pará (UFPA), Belém, Pará, Brasil. 55. Dr. Luiz Everson da Silva, Universidade Federal do Paraná (UFPR), Matinhos, Paraná, Brasil.

56. Dr. Marcelo Franco Leão, Instituto Federal de Mato Grosso (IFMT), Confresa, Mato Grosso, Brasil.

57. Dr. Marcos Guilherme Moura Silva, Universidade Federal do Pará (UFPA), Belém, Pará, Brasil.

58. Dr. Marcus Eduardo Maciel Ribeiro, Instituto Federal do Rio Grande do Sul (IFRS), Novo Hamburgo, Rio Grande do Sul, Brasil.

59. Dra. Maria Clara Silva-Forsberg, Universidade do Estado do Amazonas (UEA), Manaus, Amazonas, Brasil.

60. Me. Maria do Carmo Alves da Cruz, Universidade Federal do Maranhão (UFMA), São Luís, Maranhão, Brasil.

61. Dra. Mariuce Campos de Moraes, Universidade Federal de Mato Grosso (UFMT), Cuiabá, Mato Grosso, Brasil.

62. Dra. Maud Rejane Souza, Universidade do Estado do Amazonas (UEA), Manaus, Amazonas, Brasil.

63. Dr. Milton Rosa, Universidade Federal de Ouro Preto (UFOP), Ouro Preto, Minas Gerais, Brasil.

64. Dr. Nerio Aparecido Cardoso, Universidade Federal de Rondônia (UNIR), Porto Velho, Rondônia, Brasil.

65. Me. Patrik Marques dos Santos, Instituto Federal do Amazonas (IFAM), Parintins, Amazonas, Brasil.

66. Dr. Paulo Vilhena da Silva, Universidade Federal do Pará (UFPA), Belém, Pará, Brasil. 67. Me. Pedro Alves Fontes Neto, Instituto Federal do Maranhão (IFMA), São Luís, Maranhão, Brasil.

68. Dr. Pedro Franco Sá, Universidade do Estado do Pará (UEPA), Belém, Pará, Brasil.

69. Dr. Pedro Mathias Miranda, Universidade Federal do Acre (UFAC), Rio Branco, Acre, Brasil.

70. Me. Polyanna Possani da Costa Petry, Universidade do Estado de Mato Grosso (UNEMAT), Sinop, Mato Grosso, Brasil.

71. Me. Raquel Weyh Dattein, Instituto Estadual de Educação Osmar Poppe (IEEOP), São Luís Gonzaga, Rio Grande do Sul, Brasil. 
72. Dra. Roseli Araujo Barros, Universidade Estadual de Goiás (UEG), Jussara, Goiás, Brasil.

73. Dra. Roseli Pacheco Schnetzler, Universidade Federal do Pará (UFPA), Belém, Pará, Brasil.

74. Me. Rudinei Alves dos Santos, Instituto Federal do Pará (IFPA), Santarém, Pará, Brasil

75. Dra. Tathiana Moreira Diniz Ribeiro Cotta, Universidade do Estado do Amazonas (UEA), Manaus, Amazonas.

76. Dra. Terezinha Valim Oliver Gonçalves, Universidade Federal do Pará (UFPA), Belém, Pará, Brasil.

77. Me. Thálita Maria Francisco da Silva, Universidade Federal de Goiás (UFG), Goiânia, Goiás, Brasil.

78. Me. Uiara Mendes Ferraz de Pinho, Instituto Federal do Acre (IFAC), Rio Branco, Acre, Brasil.

79. Dr. Valdomiro Pinheiro Teixeira Junior, Universidade Federal do Sul e Sudeste do Pará (UNIFESSPA), Marabá, Pará, Brasil.

80. Me. Virgílio Bandeira do Nascimento Filho, Universidade do Estado do Amazonas (UEA), Parintins, Amazonas, Brasil.

81. Dr. Wagner Rodrigues Valente, Universidade Federal de São Paulo (UNIFESP), Guarulhos, São Paulo, Brasil.

82. Dr. Wellington Lima Cedro, Universidade Federal de Goiás (UFG), Goiânia, Goiás, Brasil.

83. Dra. Wilma de Nazaré Baía Coelho, Universidade Federal do Pará (UFPA), Belém, Pará, Brasil.

\section{Instituição Editora}

Universidade Federal de Mato Grosso (UFMT), Cuiabá, Mato Grosso, Brasil. 